\title{
Análise de modelos e práticas de planejamento e controle da produção na indústria farmacêutica
}

\author{
Marco Aurélio de Mesquita, Prof. Dr. \\ Depto. de Engenharia de Produção da Escola Politécnica da USP \\ E-mail: marco.mesquita@poli.usp.br \\ Miguel Cezar Santoro, Prof. Dr. \\ Depto. de Engenharia de Produção da Escola Politécnica da USP \\ E-mail: santoro@usp.br
}

\begin{abstract}
Resumo
O presente artigo aborda a questão da baixa utilização de modelos analíticos nos processos de decisão em planejamento da produção e estoques nas empresas. Para identificar as possíveis razões deste fato, foi realizado um estudo de caso em empresas da Indústria Farmacêutica Brasileira, por meio de visitas e entrevistas com os responsáveis pelo planejamento da produção. As empresas consideradas são multinacionais de grande porte, localizadas na região metropolitana de São Paulo. A partir dos resultados das pesquisas de campo e bibliográfica, verificou-se que a complexidade matemática e a falta de experiência dos profissionais com o desenvolvimento e implantação de modelos analíticos são os principais fatores que explicam a baixa utilização destes modelos nas empresas. Finalmente, os autores propõem algumas ações referentes a: ij formação profissional; iij parcerias com empresas de tecnologia de informação; iii) pesquisa acadêmica na área de planejamento da produção e estoques.
\end{abstract}

Palavras-chave

Pesquisa operacional, planejamento e controle da produção, MRP, teoria e prática.

\section{Analysis of models and practices in production and inventory management: a case study in Brazilian pharmaceutical industry}

\begin{abstract}
In this paper the reader will find a descriptive research, which aims to identify the reasons why people don't use operations research models in the production and inventory management. A case study is conducted in the Brazilian Pharmaceutical Industry by means of personal interviews with the operations managers of some sampled multinational companies. The results of the field research and the literature review show that both the complexity and the lack of experience with the operations research approach prevent the development and use of decision support systems based on more sophisticated models. Finally, in order to bridge the gap between theory and practice in the production and inventory management field, the authors suggest several actions in the following key areas: is the professional education; ii] the partnership between academics and the IT companies; iiij the academic research in the field of production and inventory management.
\end{abstract}

Key words

Operations research, production planning, MRP, theory and practice. 


\section{INTRODUĈ̣̃O}

Em 1996, a IFORS - The International Federation of Operational Research Societies, entidade que reúne as principais sociedades de Pesquisa Operacional, promoveu sua $14^{\text {a }}$ Conferência Internacional com o tema: "Operations Research - Bridging the Theory and Practice of Decision Making". A escolha deste tema para a conferência evidencia uma preocupação da comunidade acadêmica com respeito às dificuldades e limitações, por parte das organizações, na utilização de uma vasta gama de conhecimentos da Pesquisa Operacional. Esta preocupação está expressa também em artigos como, por exemplo, Meredith (2001), Reisman; Kirschnick (1994), Nemhauser (1994) e Geoffrion (1992).

Na Engenharia de Produção, as áreas de Planejamento e Controle da Produção e Logística são, tradicionalmente, as que apresentam maior potencial para o desenvolvimento e aplicação de modelos de otimização. Johnson; Montgomery (1974) e Hax; Candea (1984) constituem duas referências clássicas sobre a modelagem matemática dos problemas de planejamento, programação e controle da produção. Graves et al. (1993) reúnem diferentes trabalhos, onde se discutem os problemas de otimização da produção e estoques, incluindo os casos mais complexos com múltiplos produtos e estágios de produção.

Freqüentemente, encontram-se na literatura acadêmica, artigos divulgando resultados expressivos obtidos por empresas e outras organizações com a utilização de modelos de otimização. Em especial, a revista Interfaces, editada pela INFORMS - Institute for Operations Research and Management Science, tem como linha editorial promover a publicação de artigos que descrevem a aplicação de modelos de otimização em casos reais. Lin et al. (2000), Camm et al. (1997), Leachman et al. (1996), Arntzen et al. (1995) e Lee et al. (1993) podem ser citados como exemplos de destaque na aplicação de modelos de otimização na gestão da produção e estoques em empresas de manufatura.

Por outro lado, sabe-se que a utilização de modelos analíticos de apoio à decisão, nos processos de planejamento da produção e estoques em empresas, é ainda incipiente. Sanders; Manrodt (1994) fazem um levantamento sobre as práticas de previsão de demanda em empresas norte-americanas e constatam que a maioria delas não emprega técnicas estatísticas na previsão de demanda. Buxey (1995) analisa a divergência entre teoria e prática de planejamento da produção, a partir de uma pesquisa com trinta empresas australianas. Conclusões semelhantes, acerca da adequação dos modelos de planejamento agregado, podem ser encontradas no estudo de Nam; Logendran (1992).
Dentre as linhas de pesquisa ligadas ao Planejamento da Produção e Estoques, a Teoria de Programação (Scheduling Theory) é aquela que, provavelmente, reúne os maiores desafios e volume de produção científica no campo da Pesquisa Operacional. Contraditoriamente, é nesta área também que se observam as maiores dificuldades de aplicação prática. Wiers (1997), Maccarthy; Liu (1993), Dudek et al. (1992), Buxey (1989), Mckay et al. (1988) e Graves (1981) discutem os problemas de programação da produção e as razões para a baixa inserção dos modelos de otimização na indústria.

Pacheco (1999) aborda este tema no contexto brasileiro, a partir de um estudo de casos em empresas que, segundo critérios definidos pelo autor, deveriam investir na melhoria dos processos de programação da produção. Apesar da importância estratégica da programação da produção, constatou-se que o emprego de modelos clássicos de otimização é muito pouco difundido no conjunto de empresas considerado. Maccarthy; Fernandes (2000) apresentam uma classificação dos sistemas de produção que contribui para uma melhor compreensão dos problemas e conseqüente aplicação de modelos de apoio à decisão em planejamento e programação da produção.

Uma abordagem alternativa para os problemas de planejamento da produção, bastante difundida nas empresas, está associada ao modelo MRP - Material Requirements Planning, criado nos Estados Unidos no final dos anos 60 e início dos 70. O modelo auxilia na execução da produção, determinando as necessidades de materiais conforme a estrutura dos produtos que se deseja produzir, tempos de resposta (ou lead times) e as quantidades em estoque. A segunda geração do MRP, denominada MRPII - Manufacturing Resources Planning, desenvolvida na década de 80 , passou a incorporar, além das necessidades de materiais, análises de restrições de capacidade da fábrica e recursos financeiros da empresa.

Segundo Plossl (1994), a difusão do conceito MRP entre as empresas norte-americanas deve-se, em especial, ao apoio dado pela APICS - American Production and Inventory Control Society, que, desde os anos 60, promove congressos e publicações em Gestão da Produção e Estoques. Os sistemas MRPII evoluíram, na década de 90, para os sistemas integrados de gestão empresarial conhecidos como ERP - Enterprise Resources Planning. Estes sistemas têm por objetivo integrar toda a base de dados da empresa em um único banco de dados, utilizando uma arquitetura cliente-servidor. Devido a sua abrangência, que se estende por diferentes áreas da empresa (Contabilidade, Vendas, Produção, Engenharia etc.), e conseqüente complexidade, estes sistemas são constituídos de módulos que, integrados, compõem o sistema de 
gestão empresarial. Nos módulos de planejamento e programação da produção, normalmente utiliza-se a lógica do MRPII. Apesar da origem ligada à manufatura, estes sistemas são encontrados hoje em diferentes tipos de empresa, incluindo empresas do setor de serviços e financeiro.

Com a crescente difusão dos sistemas MRP na indústria, inicia-se um processo de distanciamento da pesquisa acadêmica em relação às práticas das empresas na área de planejamento da produção. Partindo-se do modelo MRP, os problemas de dimensionamento de lotes, elaboração do programa-mestre e seqüenciamento da produção são os que oferecem maiores oportunidades para incorporação de técnicas de otimização. Em Baker (1993), encontra-se uma descrição da lógica MRPII e de diferentes modelos de otimização para o dimensionamento de lotes de produção. Robinson; Dilts (1999) defendem que as pesquisas acadêmicas futuras sejam cada vez mais orientadas para a integração dos modelos de otimização com os sistemas ERP, visto que o número de empresas que implantaram este tipo de sistema é bastante expressivo e tende a crescer.

Em princípio, a maior disponibilidade de dados e de recursos computacionais nas empresas torna mais fácil o desenvolvimento e a utilização de modelos analíticos de apoio à decisão. Embora o foco original dos fornecedores de sistemas ERP fosse a racionalização do fluxo de informações (sistemas transacionais), atualmente, encontram-se disponíveis no mercado de software sistemas de apoio à decisão, genericamente denominados Advanced Planning and Scheduling - APS, voltados para solução de problemas de otimização presentes no processo de gestão da produção (SIMCHI-LEVI et al., 2000). Um aspecto que merece destaque é entender como as empresas avaliam a adequação dos sistemas disponíveis e como os utilizam no processo de planejamento da produção e estoques.

Neste trabalho, serão analisadas as práticas de planejamento da produção e estoques em um dado conjunto de empresas, focalizando, em especial, a utilização de modelos analíticos de apoio à decisão. Para efeito desta pesquisa, decidiu-se trabalhar com a indústria farmacêutica, em virtude das seguintes características: a) representam um setor industrial importante; b) constituem-se, em sua maioria, de sistema de produção intermitente para estoque (MTS - "make to stock"), com uma complexa rede de distribuição; c) em geral, são empresas de grande porte, com capacidade de investimentos em sistemas mais sofisticados de planejamento da produção e estoques.

Espera-se, com esta pesquisa, contribuir para a melhor compreensão das dificuldades e limitações associadas à utilização dos modelos matemáticos e apresentar sugestões para redução do hiato entre a teoria acadêmica e as práticas empresariais em gestão da produção e estoques, no setor industrial em questão.

\section{FORMULAC̣ÃO DO PROBLEMA}

Esta pesquisa tem como objetivo central responder a seguinte questão:

Por quê as empresas não utilizam métodos estatísticos e modelos de otimização nos processos de planejamento, programação e controle da produção e estoques?

O problema central da pesquisa pode ser desdobrado nos seguintes subproblemas: i) levantar as práticas de previsão de demanda, controle de estoques, planejamento e programação da produção; ii) identificar as expectativas das empresas e as barreiras para a introdução de modelos analíticos de apoio à decisão no planejamento da produção e estoques; iii) apresentar uma análise global e sugestões que contribuam para a difusão destes modelos. Conforme Leedy (2000), definido o problema, o passo seguinte consiste na formulação de hipóteses que, a priori, poderiam resolver o problema proposto e que servem para orientar as etapas subseqüentes do projeto de pesquisa. Para responder à questão central da pesquisa e alcançar os objetivos propostos, são formuladas três hipóteses, apresentadas abaixo.

Hipótese 1 (Capacitação): as empresas não dispõem de pessoal capacitado para o desenvolvimento e implantação de modelos analíticos de apoio à decisão.

Hipótese 2 (Adequação): os modelos analíticos disponíveis não são adequados às necessidades das empresas por: i) falta de aderência; ii) complexidade; iii) dificuldade de operacionalização.

Entende-se por falta de aderência a possibilidade de os modelos existentes não representarem satisfatoriamente os problemas reais de planejamento e programação da produção. O segundo ponto, sobre a complexidade dos modelos, consiste em afirmar que os modelos analíticos de apoio à decisão são muito complexos e de difícil compreensão para os tomadores de decisão. Por fim, a dificuldade de operacionalização diz respeito à efetiva implantação destes modelos nas empresas, que passa pelo desenvolvimento ou aquisição de softwares e a manutenção de uma base de dados confiável, resultando em um efetivo sistema de apoio à decisão para o planejamento da produção e estoques.

Hipótese 3 (Relevância): há uma percepção nas empresas de que os modelos analíticos de apoio à decisão 
disponíveis não são capazes de produzir melhorias significativas nos processos de planejamento da produção e estoques.

\section{MÉTODO DA PESQUISA}

O projeto de pesquisa que se desenvolve neste trabalho constitui uma pesquisa descritiva, que tem como objeto de estudo a Indústria Farmacêutica no Brasil. Inicia-se com um levantamento bibliográfico sobre os principais modelos de planejamento da produção e estoques e a aplicação destes na indústria. $\mathrm{O}$ passo seguinte consiste na coleta de dados, realizada a partir de entrevistas nas empresas, com o intuito de verificar a validade das hipóteses formuladas e, por decorrência, apresentar uma resposta à questão central da pesquisa.

Cogitou-se, inicialmente, que o levantamento fosse feito por questionários enviados pelo Correio, o que talvez permitisse acessar um conjunto maior de empresas. Por outro lado, a presença do entrevistador na empresa propicia uma melhor compreensão das questões e respostas do entrevistado. Além disso, corre-se o risco de um retorno muito baixo dos questionários enviados. Por estas razões, optou-se pelas entrevistas com os responsáveis diretos pelo Planejamento da Produção em algumas das maiores empresas do setor.

Este trabalho parte da premissa de que as empresas do setor buscam aumentar sua eficiência operacional e que a racionalização dos processos de planejamento da produção e estoques é parte fundamental neste processo. Além disso, em virtude dos grandes investimentos realizados em tecnologia de informação, considera-se que as empresas em questão possuem capacidade de investimento para desenvolvimento ou aquisição de softwares com modelos de apoio à decisão, caso julguem necessário.

A pesquisa concentra-se nos processos de planejamento e programação da produção e estoques (níveis tático e operacional). Questões referentes ao planejamento estratégico e da capacidade, organização da produção, tecnologia de informação e logística de suprimentos e distribuição, ainda que tenham impacto no processo de planejamento, não são tratadas explicitamente neste trabalho.

\section{A INDÚSTRIA FARMACÊUTICA NO BRASIL}

A indústria farmacêutica, responsável pela produção de medicamentos e outros produtos ligados à saúde humana, representa um setor industrial importante mundialmente, tanto do ponto de vista econômico, quanto do social, em virtude da característica dos produtos que disponibiliza para a sociedade. Estes produtos podem ser classificados em: i) medicamentos com venda sobre prescrição médica (healthcare); ii) medicamentos de automedicação responsável (consumer health); iii) produtos de consumo hospitalar e laboratorial. No primeiro grupo, há uma subdivisão entre medicamentos protegidos ou não por patente.

A Figura 1 representa as etapas do processo de produção e distribuição de medicamentos, que envolve os seguintes estágios: i) produção industrial dos fármacos (indústria química/farmacêutica); ii) produção industrial dos medicamentos (laboratórios farmacêuticos); iii) distribuição dos medicamentos para os pontos-de-venda (farmácias) através dos distribuidores de medicamentos. As etapas (i) e (ii), no caso das grandes empresas multinacionais e medicamentos protegidos por patente, estão normalmente reunidas no mesmo grupo empresarial, ou seja, os princípios ativos dos medicamentos são fornecidos por uma fábrica do mesmo grupo.

A primeira etapa da cadeia produtiva consiste na fabricação do princípio ativo, que são as substâncias responsáveis pela ação terapêutica dos medicamentos. Estas substâncias constituem o principal insumo dos laboratórios na produção de medicamentos. Os medicamentos, grosso modo, são classificados em líquidos e sólidos. Além do princípio ativo, são utilizados outros insumos e material de acondicionamento e embalagem. Cada família de produto pode ser disponibilizada comercialmente em diferentes apresentações, de acordo com a dosagem, quantidade de medicamento etc.

Na composição dos custos de produção de um medicamento, além dos custos de fabricação e logística, somam-se os custos de pesquisa e desenvolvimento dos fármacos, que são usualmente patenteados. Durante a vigência da patente, a empresa que detém o direito de exclusividade na produção do fármaco, além

Figura 1: Cadeia Produtiva da Produção de Medicamentos.

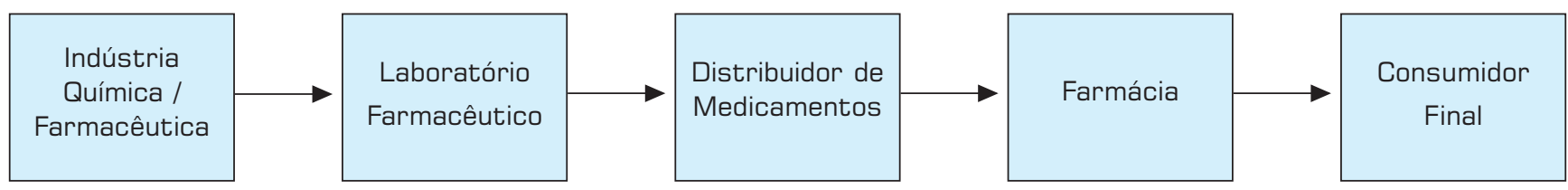


de recuperar o investimento em pesquisa e desenvolvimento, deve aproveitar para consolidar a "marca" do medicamento no mercado. Vencida a patente, empresas concorrentes podem lançar medicamentos similares no mercado.

Uma peculiaridade do processo de comercialização de medicamentos no Brasil foi a recente introdução dos medicamentos genéricos. Estes medicamentos, em geral produzidos por laboratórios concorrentes menores, apresentam o mesmo princípio ativo do produto inovador, que teve sua patente vencida. A princípio, a disseminação dos medicamentos genéricos deve provocar um aumento da competição entre os fornecedores em determinados segmentos e uma conseqüente pressão por redução de preços. Neste cenário, as funções de planejamento da produção e logística desempenham um papel similar a em outras indústrias de produtos de consumo, ou seja, são responsáveis pela redução de custos de produção e aumento da margem de lucro das empresas.

Devido às características muito particulares da indústria farmacêutica, este é um setor que nos diferentes países é bastante regulamentado pelo governo. Além dos controles e exigências para o desenvolvimento e introdução de novos produtos, os governos podem intervir também no sentido de regular as condições do próprio mercado. Existem diversas formas de atuação governamental no mercado de medicamentos, como, por exemplo, aquisição e distribuição de medicamentos para população de baixa renda, licenciamento compulsório para fabricação de medicamentos protegidos por patente, quando os preços praticados pelo laboratório que detém a patente são considerados abusivos, tabelamento de preços de medicamentos etc.
Na década de 90, em função do aumento dos custos de desenvolvimento de novos medicamentos, observou-se na indústria farmacêutica mundial um movimento de fusão de grandes empresas. Dentre as mais recentes destacam-se: i) Ciba-Geigy e Sandoz, dando origem à Novartis (Suíça); ii) Rhone-Poulenc (França) e Hoechst (Alemanha), formando a Aventis, entre outras. Estas fusões permitem, além do aumento da capacidade de investimento em pesquisa e desenvolvimento de produtos, ganhos de escala na produção e distribuição de medicamentos. Verifica-se, de forma similar a outros setores econômicos, uma reestruturação das cadeias produtivas com o surgimento de fábricas mundiais, abastecendo diferentes mercados regionais.

As principais empresas que atuam no Brasil são apresentadas na Tabela 1, que também revela a predominância dos laboratórios multinacionais (norte-americanos e europeus). Estes laboratórios estão também entre os maiores do cenário mundial.

Embora estejam instalados no Brasil grandes fabricantes mundiais de medicamentos, observa-se, de forma geral, que os laboratórios multinacionais adquirem os fármacos necessários à fabricação dos medicamentos de suas matrizes ou outras fábricas do grupo no exterior. Além da importação dos fármacos, verifica-se também um aumento da importação de medicamentos prontos e embalados. Atualmente, a importação de insumos e medicamentos pelas multinacionais instaladas no Brasil tem provocado forte impacto negativo na balança comercial do país. Do ponto de vista do desenvolvimento da indústria nacional, a capacitação em pesquisa e desenvolvimento e a recuperação da capacidade de produção local de fármacos constituem pontos fundamentais.

Tabela 1: Os maiores laboratórios no Brasil, 2002.

\begin{tabular}{|l|c|}
\hline \multicolumn{1}{|c|}{ LABORATÓRIO } & FATURAMENTO (US\$ MI) \\
\hline 1 Pfizer & 261,78 \\
\hline 2 Aventis & 237,35 \\
\hline 3 Novartis & 18,30 \\
\hline 4 Aché & 155,75 \\
\hline 5 Roche & 134,31 \\
\hline 6 EMS & 128,38 \\
\hline 7 Schering Plough & 123,48 \\
\hline 8 Schering do Brasil & 122,32 \\
\hline 10 Bristol Myers Squibb & 118,60 \\
\hline
\end{tabular}

Fonte: IMS Health. 


\section{PESQUISA DE CAMPO}

Inicialmente foram visitadas três empresas, duas do setor farmacêutico e uma do setor de alimentos. Estas entrevistas iniciais orientaram o desenvolvimento do questionário e a continuidade da pesquisa de campo. Concretizada a opção pela Indústria Farmacêutica, a empresa do setor de alimentos foi descartada, juntamente com um dos laboratórios, em função da dificuldade de contato com o responsável direto pelo planejamento operacional.

A seguir, iniciou-se o contato com as demais empresas, concentrando-se naquelas de maior porte. Foram utilizadas na pesquisa entrevistas realizadas em cinco laboratórios com fábrica na região metropolitana de São Paulo. Destes, três estão entre os dez maiores apresentados na Tabela 1, e os outros dois, de porte semelhante, alcançaram faturamento superior a US\$ $50 \mathrm{mi}$, no mesmo período. Duas das empresas da amostra são de origem norte-americana e as demais, multinacionais européias.

O questionário anexo é constituído de 45 questões, distribuídas em quatro seções: i) caracterização da empresa; ii) processos de previsão de demanda e planejamento agregado (nível tático); iii) processos de programação da produção e controle de estoques (nível operacional); iv) questões complementares como, por exemplo, formação profissional, experiência com uso de modelos, inserção da área de planejamento da produção na empresa etc. Apesar de extenso, o questionário foi utilizado como roteiro das entrevistas, que duraram, em média, duas horas e meia. Uma análise qualitativa dos resultados das entrevistas nas cinco empresas estudadas encontra-se no item a seguir.

A amostra de empresas entrevistadas é formada exclusivamente por empresas multinacionais, que são responsáveis por cerca de $75 \%$ das vendas de medicamentos no país. Apesar do tamanho reduzido, a amostra pode ser considerada representativa, em função da uniformidade dos resultados obtidos e da importância dos laboratórios participantes.

Neste conjunto de empresas, verificou-se a predominância do uso do modelo MRP na programação da produção, em especial, como instrumento de gestão de estoques de materiais e insumos importados. A decisão de compra de fármacos importados é feita com uma antecedência típica de três meses, sendo mantidos estoques de segurança destes materiais importados suficientes para cobrir as necessidades de um a dois meses de produção. Em todas as empresas visitadas, há uma versão de sistema ERP implantada.

A previsão de demanda é destacada como atividade mais importante do planejamento da produção. A meta mensal de produção é definida a partir das previsões de demanda do mês seguinte e das quantidades em estoque de cada produto, mantendo estoques de segurança dos produtos acabados, conforme a importância do item. O seqüenciamento da produção é feito manualmente pelo gerente de produção. Indicadores de giro de estoques e falta são os mais empregados na avaliação da programação da produção. Uma prioridade comum está na adoção de algum sistema de "programação com capacidade finita", que venha auxiliar na programação da produção. Foi constatada também muito pouca familiaridade com os modelos acadêmicos de planejamento da produção e estoques. Com relação aos conhecimentos sobre os modelos MRPII, tecnologia de informação e técnicas gerenciais de gestão da produção e da cadeia de suprimentos, a situação é bastante melhor.

Apesar do desconhecimento e falta de experiência com modelos analíticos de apoio à decisão, todos os entrevistados, em maior ou menor grau, manifestaram-se interessados em implantar alguma "ferramenta" matemática, que possa contribuir no processo de planejamento da produção. Quanto a essas "ferramentas", prevaleceu a preferência pela aquisição de produtos fornecidos pelo mesmo fornecedor do sistema ERP disponível na empresa. Seja no caso de desenvolvimento por terceiros, seja na aquisição de um software pronto, a adoção dependeria da comprovação de resultados, mediante testes com dados reais da empresa. Um único entrevistado destacou também a necessidade de melhorar a capacitação dos integrantes do planejamento da produção na área de planejamento da produção e logística.

Infelizmente, as entrevistas confirmaram a existência de um distanciamento acentuado entre as práticas de planejamento no segmento de empresas considerado e as teorias apresentadas nos cursos de graduação, pós-graduação e especialização. A constatação alentadora é que, apesar das divergências, existe uma predisposição em estreitar o relacionamento com o meio acadêmico, em busca de desenvolvimento de melhores técnicas e modelos de planejamento da produção e estoques.

\section{ANÁLISE DOS RESULTADOS}

A partir da pesquisa bibliográfica e dos resultados da pesquisa de campo, pretende-se identificar as barreiras para o desenvolvimento e implantação de sistemas de apoio à decisão com base em modelos analíticos de otimização. A análise do problema é feita à luz das três hipóteses formuladas no item "Formulação do problema". No próximo item, são feitas considerações acerca do hiato entre teoria e prática e apresentadas alternativas para reduzi-lo. 
Hipótese 1: Capacitação

A pesquisa de campo revelou que os responsáveis pelo planejamento da produção e estoques nas empresas consideradas têm pouca familiaridade com os conceitos e métodos da Pesquisa Operacional. Apesar de se tratar de uma amostra muito pequena, as empresas visitadas estão entre as de maior porte do setor e, por isso, não há razões para crer que a capacitação dos profissionais nas demais seja muito diferente.

Dentre os profissionais entrevistados, apenas um tem formação em Engenharia de Produção; dois são Administradores de Empresas, um Engenheiro Químico e outro bacharel em Física; nenhum deles com pós-graduação na área de Planejamento da Produção ou Logística. Tanto os administradores quanto o engenheiro de produção afirmaram ter tido contato com as disciplinas de Pesquisa Operacional e Estatística na graduação. Verificou-se, também, que nenhum destes profissionais vivenciou uma experiência real de aplicação dos métodos de Pesquisa Operacional na solução de problemas de empresas. Apesar disso, todos manifestaram interesse em aprofundar seus conhecimentos na área e desenvolver modelos para aplicação no planejamento da produção da empresa. Em particular, o fato de nunca terem participado de um projeto de desenvolvimento e implantação de modelos de otimização e estatística, quer na empresa, quer durante a graduação, é um fator que ajuda a compreender por que não se utilizam modelos analíticos nessas empresas.

\section{Hipótese 2: Adequação}

A verificação da hipótese de adequação fica limitada ao levantamento bibliográfico e a opinião pessoal do autor, pois, na pesquisa de campo, não foram identificados casos de implantação de modelos analíticos. Para orientar a discussão, a adequação dos modelos será tratada com base em: i) aderência; ii) complexidade; iii) viabilidade operacional.

Iniciando pela aderência dos modelos, ou seja, a capacidade de representar adequadamente os problemas reais de planejamento da produção e estoques, verifica-se que estes não são plenamente satisfatórios. Em geral, a abordagem acadêmica pode ser caracterizada por uma formulação muito simples e objetiva dos problemas e por uma maior sofisticação no tratamento matemático em busca da "solução ótima".

Adicionalmente, no meio acadêmico predomina a tendência de se estudar isoladamente e de forma independente cada um dos subproblemas de planejamento da produção (previsão de demanda, estoques, planejamento agregado, programação etc.). É importante resgatar a abordagem sistêmica do problema, característica da Pesquisa Operacional, presente, por exemplo, no modelo de planejamento hierárquico proposto por Hax; Candea (1984). Deve-se buscar uma estrutura de planejamento que permita integrar os vários processos e modelos de apoio à decisão. Se possível, os objetivos representados nestes modelos devem refletir não apenas a eficiência operacional, como também objetivos ligados à administração financeira e às estratégias de competição no mercado.

Uma reflexão aprofundada sobre a modelagem dos problemas de planejamento da produção e estoques pode ser encontrada em Hopp; Spearman (2000). Estes autores consideram que os modelos acadêmicos disponíveis são insatisfatórios, o que explica sua baixa utilização nas empresas, e apresentam uma estrutura hierárquica que combina modelos analíticos para planejamento e programação da produção com métodos de controle da produção baseado no princípio de produção puxada (limitação de estoques intermediários).

Por outro lado, verificou-se que o modelo MRP está presente em todas as empresas da amostra, sinalizando a existência de um padrão na indústria farmacêutica em torno da utilização do modelo MRP. Como o modelo MRP apresenta um nível menor de abstração e uma lógica mais simples que os modelos analíticos, pode-se depreender que as empresas valorizam a simplicidade do modelo, em detrimento à sofisticação dos métodos de solução empregados. Conclusões semelhantes, em relação aos modelos de programação da produção de capacidade finita, são encontradas em Pacheco (1999).

$\mathrm{O}$ segundo aspecto refere-se à complexidade dos modelos analíticos. A ênfase no estudo dos métodos de solução (algoritmos) e a falta de experiências práticas com o uso da metodologia (modelagem e implantação em casos reais) nos cursos de graduação e pós-graduação contribuem para consolidar uma imagem de que Pesquisa Operacional e Estatística são disciplinas essencialmente teóricas, de pouca utilização prática.

Finalmente, ainda com respeito à adequação, uma barreira potencial ao uso de modelos seria a dificuldade de operacionalização dos mesmos. Para que se tenha a efetiva utilização de modelos e algoritmos no uso cotidiano da empresa, é necessário consumar o desenvolvimento do modelo em sua implementação computacional. Isto pode ser feito internamente, pela área de sistemas da empresa, ou externamente, pela aquisição de softwares prontos ou desenvolvidos por fornecedor externo (feitos sob medida para a empresa).

Do exposto anteriormente, pode-se afirmar que a adequação dos modelos, analisada sob os aspectos de aderência, complexidade e viabilidade operacional, constitui efetivamente uma barreira à implantação de modelos de apoio à decisão nas empresas em questão. Na pesquisa 
de campo, esta hipótese não pode ser avaliada com maior rigor, em virtude da inexistência de experiências com modelos analíticos de apoio à decisão na amostra.

\section{Hipótese 3: Relevância}

A terceira e última hipótese refere-se à importância da adoção de métodos estatísticos e de otimização no planejamento da produção e estoques. Em função da falta de familiaridade com os mesmos, inexistência de relatos de sucesso em empresas e dos custos e riscos de implantação, é questionável a eficácia da adoção desses modelos para a melhoria dos processos de decisão. Apesar desta possível interpretação, não houve nenhuma manifestação contumaz no sentido de que a abordagem acadêmica não seja promissora. Pelo contrário, três dos entrevistados manifestaram interesse em explorar a alternativa de implantação de modelos analíticos de apoio à decisão. Dentro do planejamento da produção e estoques, houve um consenso de que as áreas de previsão de demanda e programação da produção são as que apresentam maior potencial para implantação desta classe de modelos.

\section{CONCLUSÕES}

Embora a pesquisa tenha sido realizada com um número limitado de empresas, dada a representatividade das mesmas e a regularidade de alguns resultados, podese inferir que a utilização de modelos analíticos não é muito comum nas empresas do setor, ou seja, houve a constatação da existência de um hiato entre teoria e prática na presente pesquisa. Em lugar destes, prevalece a utilização do modelo MRP (focado na administração de materiais, principalmente no controle de estoques de matérias-primas e embalagens) e uma preocupação maior em investir na tecnologia de informação e na melhoria dos processos de previsão de demanda.

A despeito dos grandes investimentos em tecnologia de informação e da importância da inovação tecnológica que caracteriza o setor farmacêutico, verificou-se na amostra que nenhuma das empresas fez investimentos no desenvolvimento de modelos de apoio à decisão. Ademais, com base nos depoimentos, caso isto venha a acontecer no futuro, por certo será dada preferência à compra de "ferramentas" (softwares prontos) disponibilizadas pelo mesmo fornecedor ou compatíveis com o sistema ERP já implantado.

Um dos objetivos deste trabalho, além do levantamento das práticas, consiste em identificar caminhos para difundir o uso de modelos analíticos no processo de planejamento e programação da produção. A fim de estruturar melhor um conjunto de iniciativas possíveis para se alcançar esse fim, considere-se que a introdução de modelos analíticos de apoio à decisão nas empresas resulta de três alternativas não excludentes, que são: i) iniciativa interna dos profissionais de planejamento da produção; ii) aquisição de software de mercado; iii) desenvolvimento e implantação com a participação de consultoria externa.

$\mathrm{Na}$ primeira alternativa, o ponto fundamental referese à formação profissional. Para que um profissional se sinta encorajado a empreitar esta atividade por iniciativa própria, além da pressão por resultados, é fundamental que ele tenha capacitação nas áreas de planejamento da produção, modelagem matemática e computação. Além do conhecimento técnico, necessita apresentar habilidades que lhe assegurem sucesso nesta iniciativa. Como a prática de uso da metodologia de pesquisa operacional é pouco difundida nas empresas, o primeiro contato e a experiência devem ocorrer ainda durante o ensino de graduação.

Neste sentido, em lugar da ênfase nos aspectos matemáticos, comum nos cursos de Pesquisa Operacional, é importante que o aluno tenha competência para formular o problema de forma adequada, do contrário, o processo nem se inicia. Além do processo de modelagem, as demais etapas de um projeto desta natureza também requerem algumas habilidades que só se desenvolvem com a prática. Assim, recomenda-se que os cursos de graduação tenham uma ênfase maior na modelagem de problemas e que proporcionem aos alunos oportunidades de desenvolvimento de trabalhos práticos em equipe, nas condições reais das empresas. Se esta experiência inicial for bem-sucedida e se estes profissionais tiverem acesso, no processo de educação continuada, a materiais de referência (não apenas livros didáticos) e suporte profissional, pode-se gradualmente modificar a frustrante realidade atual. Um exemplo de experiência deste tipo é citado por Wouters; Donsélaar (1993).

Outra forma de facilitar a introdução de modelos analíticos de apoio à decisão é através dos fornecedores de software que desenvolvem sistemas de apoio à decisão. Atualmente, é difícil avaliar os modelos embutidos nos softwares, ocultos pelos fornecedores por razões comerciais. A cooperação entre o meio acadêmico e essas empresas deve contribuir tanto para enriquecer os produtos oferecidos no mercado, quanto para proporcionar oportunidades relevantes de pesquisa e, em decorrência, de produção de novos conhecimentos. Esta cooperação contribui também para abrir uma nova perspectiva profissional para os Engenheiros de Produção, consubstanciada na atividade de desenvolvimento de sistemas de gestão empresarial. Esta parceria com os fornecedores de software tipo ERP é considerada estratégica também por Robinson; Dilts (1999). Uma barreira para a execução 
desta parceria está no fato de que este mercado é bastante concentrado e dominado por grandes empresas multinacionais, o que limita o acesso por parte de pesquisadores e estudantes.

Por fim, a atividade de consultoria, desempenhada por profissionais ligados às empresas privadas ou mesmo do meio acadêmico, constitui um importante canal para difusão de modelos de apoio à decisão. Novamente, a aproximação entre os centros de pesquisa e as empresas deve contribuir para o desenvolvimento de pesquisas e conseqüente geração de novos conhecimentos em gestão da produção.

$\mathrm{Na}$ área de Planejamento da Produção e Logística e, em particular, da Pesquisa Operacional, acredita-se que seja importante reverter a tendência de pesquisa sobre métodos de solução de problemas clássicos em benefício da pesquisa aplicada, com estudo de caso de desenvolvimento e implantação de modelos nas organizações, incentivando, inclusive, a publicação dos resultados em periódicos acadêmicos. Recomendações semelhantes podem ser encontradas em Maccarthy et.al. (2001),
Hayes (2000) e Lee; Billington (1995). No campo estritamente acadêmico, é importante promover também a aproximação entre as áreas de Pesquisa Operacional e Tecnologia de Informação.

Em síntese, relendo os ensinamentos contidos em Ackoff (1979), a área de Pesquisa Operacional deve retomar suas origens como uma disciplina voltada à solução de problemas reais encontrados nas organizações. No caso particular do Planejamento da Produção e Logística, as abordagens da Pesquisa Operacional podem trazer contribuições significativas. Para isto, é preciso recuperar as raízes da Pesquisa Operacional e valorizar a experiência do trabalho em equipes multidisciplinares na solução dos problemas de produção.

A integração universidade-empresa, através do ensino e pesquisa, deve contribuir para a produção de novos conhecimentos e uma formação profissional de qualidade. No caso específico do Planejamento da Produção e Logística, em face ao avanço da tecnologia de informação, a reunião das competências em modelagem e implantação de sistemas é fundamental.

\section{ANEXO — ROTEIRO DE ENTREVISTA PARA PESQUISA DE CAMPO}

\section{Apresentação}

Esta pesquisa destina-se a fazer um levantamento das práticas de planejamento e programação da produção nas empresas do setor farmacêutico. A partir dos dados obtidos neste levantamento, pretende-se avaliar a distância entre a teoria e as práticas de planejamento da produção e apresentar sugestões para redução deste hiato. Para que este estudo seja bem-sucedido, é fundamental a colaboração das empresas. Vale ressaltar que os dados referentes às empresas serão mantidos em sigilo e só serão divulgados mediante autorização das mesmas. Os resultados deste estudo serão posteriormente compartilhados com os participantes. Quaisquer esclarecimentos necessários, favor entrar em contato ...

\section{Identificação da Empresa}

1. Empresa:

2. Descrição da Empresa (histórico, missão, dados etc):

3. Unidades Industriais

3.1 Quantidade de Fábricas no Brasil: 
3.2 Localização e capacidade das principais unidades industriais:

4. Produtos:

4. 1 Principais Famílias de Produtos,

A)

B)

C]

4.2 Quais os principais fatores que afetam a demanda dos produtos?

5. Quem são os principais concorrentes diretos?

6. Processo de Produção:

6. 1 Fluxo de Produção:

$\square$ Lotes, para estoque

$\square$ Lotes, sob encomenda

Processo contínuo

Outros casos:

6.2 Quais são as principais etapas do processo de produção?

7. Fornecedores:

7.1 Principais Tipos de Insumos,

$\%$ Custos

Prazos (lead times)

A]

B)

C)

7.2 Descreva sucintamente o processo de suprimento e a relação com os fornecedores.

8. Clientes:

8. 1 Como é feita a distribuição física dos produtos?

8.2 Descreva sucintamente o processo de gerenciamento de pedidos.

\section{Previsão de Demanda e Planejamento da Produção}

9. A empresa faz previsões de demanda de forma sistemática e estruturada? $\square \operatorname{Sim} \square$ Não

10. Com que freqüência são realizadas as previsões de demanda?
$\square$ Quinzenal
$\square$ Mensal
$\square$ Bimestral
$\square$ Semestral
$\square$ Outra :

11. Qual o horizonte de previsão normalmente considerado?
$\square$ Mês
$\square$ Trimestre
$\square$ Semestre
$\square$ Ano
$\square$ Outro :

12. Como são feitas as previsões de demanda na empresa?

13. Utilizam Métodos Estatísticos na previsão de demanda?

$\square \operatorname{Sim} \quad \square$ Não Por quê ? 
14. Dentre os modelos abaixo, quais são conhecidos? São efetivamente utilizados?
$\square$ Média Móvel
$\square \operatorname{Sim} \quad \square$ Não
$\square$ Suavização Exponencial Simples
$\square \operatorname{Sim} \quad \square$ Não
$\square$ Suavização Exponencial com Tendência
$\square \operatorname{Sim} \quad \square$ Não
Suavização Exponencial com Sazonalidade
$\square \operatorname{Sim} \quad \square$ Não
Regressão Linear Simples
$\square \operatorname{Sim} \square$ Não
$\square$ Regressão Linear Múltipla
$\square \operatorname{Sim} \square$ Não
$\square$ Projeção com Auto-Correlação (ARIMA)
$\square \operatorname{Sim} \square$ Não
$\square$ Modelos Qualitativos baseados em Consenso
$\square \operatorname{Sim} \quad \square$ Não

$\square$ Outros Modelos:

15. Quais as dificuldades na utilização de Modelos Estatísticos na previsão de demanda?

16. Utilizam software para previsão de demanda?

$\square \operatorname{Sim} \quad \square$ Não

Qual? Quando foi implantado? Quais as razões que levaram à aquisição deste software?

Produz resultados?

17. Quais os indicadores de erro de previsão utilizados?
$\square$ Erro Médio
$\square$ Erro Absoluto Médio
$\square$ Erro Quadrático Médio
$\square$ Erro Percentual Médio $\square$ Erro Abs. Perc. Médio
$\square$ Visualização Gráfica
$\square$ Outros:

18. Como as previsões de demanda são utilizadas no planejamento agregado da produção?

19. Qual o horizonte de planejamento da produção normalmente considerado?
$\square$ Mês
$\square$ Trimestre
$\square$ Semestre
$\square$ Ano
Outro:

20. Qual o período de revisão do planejamento?
$\square$ Mês
$\square$ Trimestre
$\square$ Semestre
$\square$ Ano
$\square$ Outro :

21. Como é feito o planejamento agregado da produção?

22. Utilizam modelo de programação linear para o planejamento agregado da produção? $\square$ Sim $\square$ Não

Há quanto tempo? Como foi implantado na empresa? Produz resultados?

23. Utilizam modelo MRP II para o planejamento da produção? $\square$ Sim $\square$ Não

Qual o software utilizado? Quando foi efetivamente implantado?

Quais as razões que levaram a empresa a adotar um modelo MRPII ? Produz resultados?

24. Utilizam Sistemas ERP? $\square$ Sim $\square$ Não

Qual? Quando foi implantado? Quais os módulos utilizados?

Trouxe benefícios significativos para a empresa? $\quad \square \operatorname{Sim} \quad \square$ Não Quais?

25. Na sua opinião, o sistema atual de planejamento da produção e estoques está adequado às necessidades da empresa? $\square$ Sim $\square$ Não Por quê?

26. Quais são as prioridades da empresa na área de planejamento e controle da produção? 


\section{Programação da Produção e Estoques}

27. Como é feita a programação mensal da produção?

28. Como são consideradas as restrições de capacidade e as necessidades de materiais?

29. Como são considerados os custos de estoque e o nível de atendimento aos clientes?

30. Como é feita a avaliação da programação da produção / reposição de estoques na empresa?

31. Utilizam modelos clássicos de estoques (estoque base, reposição periódica, etc.) para:
a) controle de matérias-primas e outros insumos?
$\square \operatorname{Sim} \quad \square$ Não
b) controle de produtos acabados na fábrica?
$\square \operatorname{Sim} \quad \square$ Não
c) controle de estoques nos centros de distribuição?
$\square \operatorname{Sim} \quad \square$ Não
d) controle de estoques nos pontos-de-venda?
$\square \operatorname{Sim} \quad \square$ Não
32. Utilizam modelo de otimização na formação dos lotes de produção?
$\square \operatorname{Sim} \square$ Não

Como foi implantado na empresa? Produz resultados?

33. Como é feita a programação detalhada (seqüenciamento) da produção?

34. Utilizam modelos de simulação para programação da produção? $\quad \square \operatorname{Sim} \quad \square$ Não Qual o software de simulação utilizado? Produz resultados?

35. Utilizam modelos matemáticos para programação da produção? $\quad \square \operatorname{Sim} \quad \square$ Não Quando foi implantado? Quais os critérios de otimização e restrições considerados? Produz resultados? Quais as dificuldades de implantação e uso deste tipo de modelo?

36. Qual o grau de familiaridade (escala de 0 a 5) com os seguintes modelos de programação matemática [Scheduling]? $\begin{array}{llllll}0 & 1 & 2 & 3 & 4 & 5\end{array}$

a) Programação Linear Inteira ("branch-and-bound']

b) Algoritmos de Otimização para uma única máquina

c) Algoritmos de Otimização para flow shop

d) Algoritmos de Otimização para job shop

e) Heurísticas de Busca para problemas combinatórios

f) Heurísticas com Regras de Seqüenciação

g) Heurísticas baseadas em Recurso Gargalo

h) Outros:

37. Como é feito o controle da produção e como este se relaciona com a programação?

38. Como as decisões de planejamento (nível tático) afetam as decisões de programação (nível operacional)? Qual o grau de integração entre o planejamento e a programação? 


\section{Tópicos Complementares}

39. Existem, na área de planejamento da produção e estoques, profissionais com conhecimentos e experiência em Pesquisa Operacional e/ou Estatística? $\square \operatorname{Sim} \quad \square$ Não

Quantos? Qual a formação destes profissionais?

40. A empresa já teve alguma experiência com o desenvolvimento de modelos matemáticos de apoio à decisão para o planejamento da produção e logística? $\square \operatorname{Sim} \quad \square$ Não

Descreva e avalie sucintamente esta[s] experiência[s].

41. Acredita que modelos de otimização e técnicas estatísticas podem contribuir para melhoria do processo de planejamento da produção e logística da empresa? $\quad \square \operatorname{Sim} \quad \square$ Não Por quê?

42. Quais as maiores dificuldades para o desenvolvimento e implantação de modelos de otimização e técnicas estatísticas no planejamento da produção em sua empresa?

43. Quais são os critérios utilizados pela empresa para avaliação e implantação de modelos de apoio a decisão na área de planejamento da produção?

44. Qual a importância relativa do planejamento da produção e logística na empresa?

45. No planejamento da produção, quais são as atividades consideradas mais relevantes: planejamento agregado, previsão, suprimento, programação, estoques ou distribuição?

Fique à vontade para fazer quaisquer sugestões ou comentários adicionais:

\section{Identificação}

Para facilitar futuros contatos e esclarecimento de dúvidas,

Nome:

Cargo:

Telefone: e-mail:

Obrigado !

Artigo recebido em 07/06/2003

Aprovado para publicação em 25/02/2004 


\section{- Referências Bibliográficas}

ACKOFF, R. The future of Operational Research is Past. Operations Research, v. 30, n. 2, p. 93-104, 1979.

ARNTZEN, B. C.; BROWN, G. G. HARRISON, T. P.; TRAFTON, L. L. Global Supply Chain Management at Digital Equipment Corporation. Interfaces, v. 25, n. 1, p. 69-93, 1995.

BAKER, K. R. Requirements Planning In: GRAVES, S. C. et al. Handbooks in Operations Research and Management Science, v. 4, Amsterdam: NorthHolland, 1993.

BUXEY, G. Production Scheduling practice and theory. European Journal for Operational Research, v. 39, p. 17-31, 1989.

BUXEY, G. A Managerial Perspective on Aggregate Planning. Internationa Journal of Production Economics, v. 41 p. 127-133, 1995.

CAMM, J. D.; CHORMAN, T. H.; DILL, F. A.; EVANS, J. R.; SWEENEY, D. J.; WEGRYN, G. W. Blending OR/MS Judgment, and GIS: restructuring P\&G's Supply Chain. Interfaces, v. 27, n. 1, p. 128-142, 1997.

DUDEK, R. A.; PANWALKAR, S. S SMITH, M. L. The Lessons of Flowshop Scheduling Research. Operations Research, v. 40, n. 1, p. 7-13, 1992.

GEOFFRION, A. M. Forces, Trends, and Opportunities in MS/OR. Operations Research, v. 40, n. 3, p. 423-445, 1992.

GRAVES, S. C. A review of Production Scheduling. Operations Research, v. 29 , n. 4 , p. $646-675,1981$.
GRAVES, S. C.; RINNOOY KAN, A. H. G.; ZIPKIN, P. H. (editores). Logistics of Production and Inventory. Amsterdam: North-Holland, 1993. (Handbooks in Operations Research and Management Science, v. 4)

HAX, A. C.; CANDEA, D. Production and Inventory Management. Nova Jersey: Prentice-Hall, 1984

HAYES, R. H. Toward a 'New Architecture' for POM. Production and Operations Management, v. 9, n. 2, p. 105-110, 2000

HOPP, W. J.; SPEARMAN, M. L. Factory Physics: foundations of manufacturing management. 2.ed., Boston: Irwin McGraw-Hill, 2000.

JOHNSON, L. A.; MONTGOMERY, D. C.: Operations Research in Production Planning, Scheduling, and Inventory Control. Nova York: Wiley, 1974.

LEACHMAN, R. C.; BENSON, R. F.; LIU, C.; RAAR, D. J. IMPReSS: an automated production-planning and deliveryquotation systems at Harris Corporation semiconductor sector. Interfaces, v. 26, n. 1, p. 6-37, 1996.

LEE, H. L.; BILLINGTON, C. The Evolution of Supply Chain Management Models and Practice at Hewlett-Packard. Interfaces, v. 25, n. 5, p. 42-63, 1995.

LEE, H. L.; BILLINGTON, C.; CARTER, B. Hewlett-Packard Gains Control of Inventory and Service through Design for Localization. Interfaces, v. 23, n. 4 , p. 1-11, 1993.

LEEDY, P. D. Practical Research: planning and design. 7.ed., Nova Jersey: Prentice-Hall, 2000
LIN, G.; ETTL, M.; BUCKLEY, S.; BAGCHI, S.; YAO, D. D.; NACCARATO, B.L.; ALLAN, R.; KIM, K.; KOENIG, L. Extended Enterprise Supply Chain Management at IBM Personal Systems Group and Other Divisions. Interfaces, v. 30, n. 1, p. 7-25, 2000.

MACCARTHY, B. L.; FERNANDES, F. C. F. A Multidimensional Classification of Production Systems for the Design and Selection of Production Planning and Control Systems. Production Planning and Control, v. 11, n. 5, p. 481496, 2000.

MACCARTHY, B. L.; LIU, J. Addressing the Gap in Scheduling Research: a review of optimization and heuristic methods in production scheduling. International Journal of Production Research, v. 31, n. 1, p. 59-79, 1993.

MACCARTHY, B. L.; MCKAY, K. N.; WAEFLER, T. Letter to the Editor. Computers in Industry, v. 44, n. 2, p. 99103, 2001.

MCKAY, K. N.; SAFAYENI, F. R ; BUZACOTT, J. A. Job-Shop Scheduling Theory: what is relevant? Interfaces, v. 18 , n. 4 , p. $84-90,1988$.

MEREDITH, J. R. Reconsidering the Philosophical Basis of OR/MS. Operations Research, v. 49, n. 3, p. 325333, 2001.

NAM, S.; LOGENDRAN, R. Aggregate Production Planning: a survey of models and methodologies. European Journal of Operational Research, v. 61, p. 255-272, 1992

NEMHAUSER, G. L. The Age of Optimization: solving large-scale real-world problems. Operations Research. v. 42, n. 1, p. 5-13, 1994.
PACHECO, R. F. A adoção de modelos de programação da produção nas empresas: um estudo do processo de decisão. 181 p. Tese (Doutorado) Escola Politécnica, Universidade de São Paulo, 1999.

PLOSSL, G. Orlicky's Material Requirements Planning. 2. ed., Nova York: McGraw-Hill, 1994.

REISMAN, A.; KIRSCHNICK, F. The devolution of OR/MS: implications from a statistical content analysis of papers in flagship journals. Operations Research. v. 42, n. 4, p. 577 588, 1994.

ROBINSON, A. G.; DILTS, D. M. OR \& ERP: a match for the new millennium? OR/MS Today. v. 26, n. 3 , 1999.

SANDERS, N. R.; MANRODT, K. B Forecasting Practices in US Corporations: Survey Results Interfaces. v. 24, n. 2, p. 92-100, 1994

SIMCHI-LEVI, D.; KAMINSKY, P.; SIMCHI-LEVI, E. Designing and Managing the Supply Chain: concepts, strategies and case studies. Boston: McGraw-Hill, 2000.

WIERS, V. C. S. A Review of the Applicability of OR and AI Scheduling Techniques in Practice. Omega. v. 25 n. 2, p. 145-153, 1997.

WOUTERS, M. J. F.; VAN DONSÉLAAR, K. H. Design of Operations Management Internships Across Organizations: learning OM by doing OM. Interfaces, v. 30 , n. 4 , p. 81-93, 1993. 